\title{
EDITORIAL
}

\section{Knowledge-based development}

Knowledge in science and technology and its management is now recognized world wide as the driving force of economic growth, leading to social well being and prosperity. Knowledge-based economies depend on production, dissemination and use of knowledge and information, particularly in science and technology. In such an economy investment in research and development, education and training as well as new managerial approaches in science and technology are crucial factors.

There are key challenges which have to be faced for science and technology to contribute to greater productivity. Foremost among these is the necessity for the science and technology policies of a country to be coherent with the national development goals. To achieve this, it is necessary for the science and technology policies and the economic policies to complement and synergise each other. The scientific and technological community which includes among others, agriculturists, engineers, industrialists, urban planners and health professionals must necessarily increase their interactions with the policy makers to ensure the formulation of development-oriented policies and strategies as well as their implementation. This interaction will result in appropriate frameworks for enhanced research and communication of research findings to decision makers. This links scientific and technological knowledge with the formulation of strategic policies and programmes. It will also enable the prioritising of research to find solutions to pressing problems in the country.

Another challenge is the weakness in the knowledge base. The socio-cultural, political and economic development influences the formulation of the appropriate policies that together affects the development of the knowledge base of a country. In addition to this the interaction and collaboration between researchers - the producers of knowledge - and industry - the users of this knowledge- also has a direct influence on the capacity of the economy to produce the products and services demanded by the changing market needs.
The issue of educational imbalances that hamper the country meeting the demands of a knowledge-based economy has also to be addressed. Among these, the regional imbalances stand out. Opportunities for the rural community to have access to higher education, particularly in science and technology need enhancement. The system of education in the country needs to lay a greater emphasis on technical education.

A lack of a critical mass of human resources in research and development, low number of research publications and patents are the important factors that have resulted in a weak knowledge base in the country. The country must improve the competitiveness of its knowledge and science and technology workers through continuous development of the human capital base. This can be achieved by improving the level of skills and knowledge of the science and technology workers. The capability of the workers to learn new skills and apply them is of paramount importance to absorb and use new technologies. Towards this end, it is necessary to build adequate capacity for absorption, assimilation and application of knowledge/technologies. Due to advances in knowledge, the relevant technologies become obsolete rapidly. It is therefore important for the workforce to engage in continuing and life long education and training. The responsibility of a knowledgeable and appropriately skilled workforce in the country has to be borne by academia, the educational institutions and the industry.

Brain Drain has also contributed significantly to the lack of a critical mass of human resources in research and development. In addition to stopping Brain Drain there must be policies that encourage its reversal whereby reputed scientists and technologists are given encouragement to return and contribute to the development efforts of the country.

Access to knowledge and technology is vital for the development of the rural and remote communities of the country. The Ministry of Science and Technology has already launched the Vidatha programme to facilitate and 
accelerate the dissemination of relevant research findings in science and technology to the rural communities. This programme provides the rural communities with a mechanism to access knowledge, link with other communities and facilitates entrepreneurship leading to their empowerment.

In levering knowledge for greater productivity and global competitiveness, appropriate technologies are identified for targeting those who can make the most effective use of such technologies. The success of such a programme depends heavily on the facilitation and development of entrepreneurship. To produce successful entrepreneurs it is necessary to encourage competency and capacity. The challenge before us then is to enable the micro, small and medium scale enterprises to become viable growing business concerns by providing technologies, avenues for credit as well as legal and marketing assistance.

The generation of knowledge is the most important factor for the progress in a knowledge-based economy. Knowledge creation requires suitable and favourable policies particularly with regard to investment in research and development. A strong partnership between researchers of both the public and private sector is a necessary condition for the creation of knowledge to upgrade technology and increase productivity.

Nalini Ratnasiri 